\title{
Ischemic and Bleeding Events in PENDULUM Patients With High Bleeding Risk and High Platelet Reactivity
}

\author{
Raisuke Iijima, MD, PhD; Kazushige Kadota, MD, PhD; Koichi Nakao, MD, PhD; \\ Yoshihisa Nakagawa, MD, PhD; Junya Shite, MD, PhD; Hiroyoshi Yokoi, MD, PhD; \\ Ken Kozuma, MD, PhD; Kengo Tanabe, MD, PhD; Takashi Akasaka, MD, PhD; \\ Toshiro Shinke, MD, PhD; Takafumi Ueno, MD, PhD; Atsushi Hirayama, MD, PhD; \\ Shiro Uemura, MD, PhD; Atsushi Harada; Takeshi Kuroda, PhD; Atsushi Takita; \\ Yoshitaka Murakami, PhD; Shigeru Saito, MD, PhD; Masato Nakamura, MD, PhD
}

\begin{abstract}
Background: The balance between thrombotic and bleeding risk is of great concern in high bleeding risk (HBR) patients. This study evaluated the relationship between perioperative antiplatelet reactivity and thrombotic and bleeding events in patients at HBR undergoing percutaneous coronary intervention (PCl).

Methods and Results: In this post hoc analysis of the PENDULUM (Platelet rEactivity in patieNts with DrUg eLUting stent and balancing risk of bleeding and ischeMic event) registry, patients undergoing PCl were categorized as HBR or non-HBR, and stratified as having high platelet reactivity (HPR; P2Y 12 reaction unit $[P R U]>208$ ) or non-HPR (PRU $\leq 208$ ). Cumulative incidences of cardiovascular and cerebrovascular events (Journal of the American College of Cardiology expert definitions) and bleeding events (Bleeding Academic Research Consortium criteria) were assessed 12 months after index PCl. The incidence of ischemic and bleeding events was $\sim 3$-fold higher in HBR vs. non-HBR patients. Thrombotic/ischemic events were significantly more common in the HPR subgroup in HBR patients (hazard ratio [HR]: 1.59; 95\% confidence interval [Cl]: 1.11-2.28; $\mathrm{P}=0.012$ ), but there was no difference in non-HBR patients. After adjustment for covariates, HPR in HBR patients remained an independent factor for thrombotic and ischemic events (HR: 1.69; 95\% Cl: 1.13-2.54; $\mathrm{P}=0.011$ ), but not for bleeding events (HR: 1.56; 95\% Cl: 0.78-3.11; $\mathrm{P}=0.210$ ).
\end{abstract}

Conclusions: Maintaining adequate PRU levels during $\mathrm{PCl}$ is an important factor in improving clinical outcomes, especially for HBR patients.

Key Words: Academic Research Consortium-high-bleeding risk (ARC-HBR); High bleeding risk; Mortality; PENDULUM registry; Percutaneous coronary intervention

$\mathbf{P}$ atients undergoing insertion of drug eluting stents commonly receive dual antiplatelet therapy (DAPT) to prevent thrombotic and ischemic complications, ${ }^{1-3}$ but some may experience fatal bleeding events as a result. Advances in stent technology, such as durable polymers, ideal drug elution, and thinner stent struts, have

\begin{tabular}{l} 
Editorial p ???? \\
\hline dramatically reduced the risk of device-associated ischemic \\
and thrombotic events. Therefore, in recent years, the focus \\
has shifted to managing bleeding complications associated
\end{tabular}

Received August 6, 2021; revised manuscript received October 8, 2021; accepted October 24, 2021; J-STAGE Advance Publication released online December 8, 2021 Time for primary review: 25 days

Division of Cardiovascular Medicine, Toho University Ohashi Medical Center, Tokyo (R.I., M.N.); Department of Cardiology, Kurashiki Central Hospital, Okayama (K. Kadota); Division of Cardiology, Saiseikai Kumamoto Hospital Cardiovascular Center, Kumamoto (K.N.); Department of Cardiovascular Medicine, Shiga University of Medical Science, Shiga (Y.N.); Division of Cardiology, Osaka Saiseikai Nakatsu Hospital, Osaka (J.S.); Cardiovascular Medicine Center, Fukuoka Sanno Hospital, Fukuoka (H.Y.); Division of Cardiology, Department of Internal Medicine, Teikyo University, Tokyo (K. Kozuma); Division of Cardiology, Mitsui Memorial Hospital, Tokyo (K.T.); Department of Cardiovascular Medicine, Wakayama Medical University, Wakayama (T.A.); Division of Cardiology, Department of Medicine, Showa University School of Medicine, Tokyo (T.S.); Department of Cardiovascular Medicine, Fukuoka Kinen Hospital, Fukuoka (T.U.); Department of Cardiology, Osaka Police Hospital, Osaka (A. Hirayama); Department of Cardiology, Kawasaki Medical School, Okayama (S.U.); Medical Information Department (A. Harada), Primary Medical Science Department (T.K.), Data Intelligence Department (A.T.), Daiichi Sankyo Co., Ltd., Tokyo; Department of Medical Statistics, School of Medicine, Toho University, Tokyo (Y.M.); and Division of Cardiology \& Catheterization Laboratories, Shonan Kamakura General Hospital, Kanagawa (S.S.), Japan

Mailing address: Raisuke Iijima, MD, PhD, Division of Cardiovascular Medicine, Toho University Ohashi Medical Center, 2-22-36 Ohashi, Meguro-ku, Tokyo 153-8515, Japan. E-mail: raisuke@oha.toho-u.ac.jp

All rights are reserved to the Japanese Circulation Society. For permissions, please e-mail: cj@j-circ.or.jp

ISSN-1346-9843 


\section{with DAPT. ${ }^{4,5}$}

To aid physicians in identifying patients at high bleeding risk (HBR), the Academic Research Consortium (ARC) published a consensus document in 2019, which provided a consistent definition of HBR to be used in clinical trials and routine practice to evaluate and standardize treatment. ${ }^{6}$ Recent investigations confirmed the applicability of ARC-HBR for identifying bleeding risk in routine clinical practice. ${ }^{7,8}$ It is anticipated that the identification of HBR patients could be a valuable indicator of the optimal duration of antiplatelet therapy after percutaneous coronary intervention (PCI) and may contribute to improved clinical outcomes.

It is well known that the risk factors for bleeding and ischemic events overlap, ${ }^{9-12}$ and that HBR patients can also be presumed to be at increased risk for fatal ischemia. Therefore, investigating the balance of risk for thrombotic and bleeding events after PCI is particularly relevant for HBR patients. However, data relating to ischemic risk and consequent prognosis in HBR patients are scarce. Among patients planned to undergo PCI, the proportion of patients with HBR approaches $39-44 \%$, and it is well known that such patients suffer from bleeding and thrombotic/ischemic events early after PCI.7,8 To enhance the efficacy and safety of antithrombotic therapy after insertion of drug-eluting stents, de-escalation strategies to reduce the risk of bleeding, as well as escalation strategies to increase efficacy, have been intensively investigated. Indeed, a recent meta-analysis suggested that selection of antiplatelet therapy should be guided by means of platelet function testing or genetic testing in patients undergoing PCI, rather than using standard regimens. ${ }^{13}$ Although this clinical challenge is of particular relevance to HBR patients, to date, no studies have explored outcomes in HBR patients compared with non-HBR patients. Therefore, we explored the relationship between platelet reactivity and post-PCI clinical events in HBR patients, anticipating that this evaluation may provide deeper insights for determining treatment strategies for this difficult-to-treat patient population.

In this analysis, we evaluated outcomes according to the new Journal of the American College of Cardiology (JACC) expert definition of thrombotic and ischemic events, ${ }^{14}$ and assessed the results with respect to the traditional definition of a major adverse cardiac and cerebrovascular event (MACCE). As conventional MACCE outcomes include bleeding-related events, which may misrepresent the true efficacy and safety of antithrombotic therapy, we applied the JACC expert recommendations to gain a more accurate assessment of the association between antiplatelet effects and clinical events in HBR patients.

\section{Methods}

\section{Patients}

Detailed inclusion and exclusion criteria for the PENDULUM Registry have been published. ${ }^{15}$ In brief, patients aged $\geq 20$ years at the time of informed consent, with coronary artery lesions that were visually confirmed by coronary angiography and for which PCI was indicated by the placement of a second-generation drug-eluting stent, and who received antiplatelet drugs, were enrolled. All patients provided written informed consent before study inclusion. To reduce potential bias, all patients who provided informed consent were registered consecutively.

\section{Study Design}

PENDULUM was a multicenter, prospective registry of Japanese patients who underwent PCI in the real-world setting. ${ }^{15,16}$ The study was registered in the University hospital Medical Information Network Clinical Trials Registry (UMIN-CTR), with the identifier, UMIN000020332. The study protocol and related documents were approved by the Ethics Committee at Toho University Ohashi Medical Center on 14 December 2015 (reference: 15-71). The study was performed in accordance with the principles of the Declaration of Helsinki and the International Council for Harmonization Good Clinical Practice Guidelines.

Between December 2015 and June 2017, patients from 67 centers in Japan were enrolled. There was no mandated study treatment. The choice of antiplatelet drug and the dosage administered to patients was made at the investigator's discretion. Furthermore, the treatment of any complications that arose during follow up was not subject to any limitations.

This article describes the results of a post hoc analysis of data from the PENDULUM (Platelet rEactivity in patieNts with DrUg eLUting stent and balancing risk of bleeding and ischeMic event) Registry, using patients previously categorized according to ARC-HBR criteria as HBR or non-HBR. ${ }^{16}$ According to the modified ARC-HBR score, patients were allocated 1 point for each major criterion and 0.5 points for each minor criterion. Patients with a score of $\geq 1$ point were assigned to the HBR group, and patients with a score of 0 or 0.5 were categorized as non-HBR.

\section{Outcomes}

Endpoints evaluated in this analysis included the cumulative incidences of cardiovascular and cerebrovascular events, and bleeding events, 12 months after the index PCI, according to HBR status. Cardiovascular and cerebrovascular events were assessed using the new JACC expert definition of thrombotic/ischemic events ${ }^{\mathbf{1 4}}$ (comprising cardiovascular death, non-fatal myocardial infarction [MI], non-fatal ischemic stroke) and using MACCE (the standard clinical definition comprising all-cause death, non-fatal MI, nonfatal stroke, and stent thrombosis) (Supplementary Table 1). The occurrence of the first major bleeding event and cumulative rates after 12 months were also calculated. Bleeding was defined according to the Bleeding Academic Research Consortium (BARC) criteria. ${ }^{17}$ Major bleeding was defined as BARC types 3 or 5 bleeding.

Outcomes were also assessed according to platelet reactivity. Using a P2Y 12 reaction unit (PRU) value of 208 as the cut-off, high platelet reactivity (HPR) was classified as PRU $>208$ and non-HPR as PRU $\leq 208,{ }^{18}$ measured between 12 and $48 \mathrm{~h}$ post-PCI.

Other outcomes evaluated in this analysis included the cumulative incidence of net adverse clinical events (NACE) at 12 months after PCI, net clinical benefit (JACC expert definition ${ }^{\mathbf{1 4}}$ ) (Supplementary Table 1), individual event rates at 12 months after PCI, and determination of independent correlates of mortality following ischemic events.

\section{Statistical Methods}

The sample size used has already been discussed in a previous paper. ${ }^{15}$ For this post hoc analysis, continuous variables were reported as mean \pm standard deviation (SD). Categorical data were reported as proportions. For the comparison of baseline characteristics between HPR and non-HPR subgroups among patients with and without 


\begin{tabular}{|c|c|c|c|c|c|c|c|}
\hline & \multirow{2}{*}{$\begin{array}{c}\text { All } \\
(\mathrm{N}=6,267)\end{array}$} & \multicolumn{3}{|c|}{ HBR } & \multicolumn{3}{|c|}{ Non-HBR } \\
\hline & & $\begin{array}{c}\text { HPR } \\
(n=1,408)\end{array}$ & $\begin{array}{l}\text { Non-HPR } \\
(n=1,605)\end{array}$ & $P$ value & $\begin{array}{c}\text { HPR } \\
(n=819)\end{array}$ & $\begin{array}{l}\text { Non-HPR } \\
(n=2,074)\end{array}$ & $P$ value \\
\hline Age, years & $70.0 \pm 10.7$ & $74.9 \pm 9.2$ & $74.9 \pm 9.1$ & 0.988 & $66.6 \pm 9.5$ & $64.4 \pm 10.0$ & $<0.001$ \\
\hline$\geq 75$ & $2,324(37.1)$ & $854(60.7)$ & $965(60.1)$ & - & $135(16.5)$ & 241 (11.6) & - \\
\hline Sex, male & 4,909 (78.3) & $993(70.5)$ & $1,209(75.3)$ & 0.003 & $657(80.2)$ & $1,770(85.3)$ & $<0.001$ \\
\hline Body weight, kg & $64.0 \pm 12.6$ & $60.8 \pm 11.6$ & $61.0 \pm 12.5$ & 0.767 & $66.4 \pm 12.8$ & $67.6 \pm 12.2$ & 0.022 \\
\hline$\leq 50$ & 794 (12.7) & $251(17.8)$ & $298(18.6)$ & 0.591 & $69(8.4)$ & $127(6.1)$ & 0.029 \\
\hline BMI, $\mathbf{k g} / \mathrm{m}^{2}$ & $24.2 \pm 3.6$ & $23.8 \pm 3.5$ & $23.6 \pm 3.6$ & 0.088 & $24.7 \pm 3.7$ & $24.8 \pm 3.5$ & 0.248 \\
\hline \multicolumn{8}{|l|}{ Medical history } \\
\hline Hypertension & $5,186(82.8)$ & $1,251(88.8)$ & $1,381(86.0)$ & 0.021 & 654 (79.9) & $1,606(77.4)$ & 0.156 \\
\hline Hyperlipidemia & $4,919(78.5)$ & $1,117(79.3)$ & $1,145(71.3)$ & $<0.001$ & $678(82.8)$ & 1,698 (81.9) & 0.564 \\
\hline Diabetes mellitus & $2,767(44.2)$ & 717 (50.9) & $711(44.3)$ & $<0.001$ & $358(43.7)$ & $827(39.9)$ & 0.059 \\
\hline Current smoker & 1,327 (21.2) & 177 (12.6) & 245 (15.3) & 0.068 & $232(28.3)$ & $581(28.0)$ & 0.851 \\
\hline Heart failure & $850(13.6)$ & 314 (22.3) & $295(18.4)$ & 0.013 & $57(7.0)$ & $137(6.6)$ & 0.356 \\
\hline Peripheral artery disease & $421(6.7)$ & $178(12.6)$ & $128(8.0)$ & $<0.001$ & $34(4.2)$ & $59(2.8)$ & 0.073 \\
\hline Atrial fibrillation & $538(8.6)$ & $208(14.8)$ & $248(15.5)$ & 0.670 & $19(2.3)$ & $40(1.9)$ & 0.747 \\
\hline Malignancy & $367(5.9)$ & $160(11.4)$ & $190(11.8)$ & 0.685 & $0(0.0)$ & $0(0.0)$ & - \\
\hline History of MI & $1,575(25.1)$ & $393(27.9)$ & $390(24.3)$ & 0.026 & $226(27.6)$ & $489(23.6)$ & 0.021 \\
\hline History of $\mathrm{PCl}$ & $2,567(41.0)$ & $669(47.5)$ & $626(39.0)$ & $<0.001$ & $374(45.7)$ & 765 (36.9) & $<0.001$ \\
\hline History of CABG & $265(4.2)$ & $77(5.5)$ & $95(5.9)$ & 0.595 & $23(2.8)$ & $59(2.8)$ & 0.958 \\
\hline History of ischemic stroke & $655(10.5)$ & $266(18.9)$ & $265(16.5)$ & 0.060 & $36(4.4)$ & $59(2.8)$ & 0.028 \\
\hline History of TIA & $80(1.3)$ & $27(1.9)$ & $28(1.7)$ & 0.656 & $11(1.3)$ & $12(0.6)$ & 0.034 \\
\hline History of intracranial bleeding & $124(2.0)$ & $54(3.8)$ & $64(4.0)$ & 0.930 & $0(0.0)$ & $0(0.0)$ & - \\
\hline History of GI bleeding & $183(2.9)$ & $76(5.4)$ & $72(4.5)$ & 0.179 & $4(0.5)$ & $16(0.8)$ & 0.419 \\
\hline \multicolumn{8}{|l|}{ Clinical presentation } \\
\hline Non-ACS & $4,252(67.8)$ & $1,047(74.4)$ & $1,104(68.8)$ & \multirow{2}{*}{$<0.001$} & $560(68.4)$ & $1,336(64.4)$ & \multirow{2}{*}{0.044} \\
\hline ACS & 2,015 (32.2) & $361(25.6)$ & $501(31.2)$ & & $259(31.6)$ & $738(35.6)$ & \\
\hline Unstable angina & $790(12.6)$ & $150(10.7)$ & $214(13.3)$ & 0.024 & $75(9.2)$ & $296(14.3)$ & $<0.001$ \\
\hline Non-STEMI & $323(5.2)$ & $69(4.9)$ & $89(5.5)$ & \multirow{2}{*}{0.696} & $29(3.5)$ & $111(5.4)$ & \multirow{2}{*}{0.012} \\
\hline STEMI & $908(14.5)$ & $143(10.2)$ & $199(12.4)$ & & $155(18.9)$ & $334(16.1)$ & \\
\hline \multicolumn{8}{|l|}{ Medication at discharge } \\
\hline Thienopyridine & $6,195(98.9)$ & $1,376(97.7)$ & $1,587(98.9)$ & 0.012 & $807(98.5)$ & $2,070(99.8)$ & $<0.001$ \\
\hline Clopidogrel & $2,213(35.3)$ & $791(56.2)$ & $479(29.8)$ & \multirow{2}{*}{$<0.001$} & $350(42.7)$ & $471(22.7)$ & \multirow{2}{*}{$<0.001$} \\
\hline Prasugrel & $3,921(62.6)$ & $561(39.8)$ & $1,093(68.1)$ & & $451(55.1)$ & $1,585(76.4)$ & \\
\hline Aspirin & $6,143(98.0)$ & $1,370(97.3)$ & $1,558(97.1)$ & 0.704 & $811(99.0)$ & $2,052(98.9)$ & 0.841 \\
\hline OAC & $610(9.7)$ & $250(17.8)$ & $342(21.3)$ & 0.014 & $0(0.0)$ & $0(0.0)$ & - \\
\hline PPI & $5,295(84.5)$ & $1,197(85.0)$ & $1,334(83.1)$ & 0.156 & $713(87.1)$ & $1,744(84.1)$ & 0.044 \\
\hline NSAIDs except aspirin & $334(5.3)$ & $112(8.0)$ & $132(8.2)$ & 0.788 & $19(2.3)$ & $53(2.6)$ & 0.719 \\
\hline Steroids & $250(4.0)$ & $79(5.6)$ & $109(6.8)$ & 0.182 & $12(1.5)$ & $37(1.8)$ & 0.551 \\
\hline
\end{tabular}

Data are presented as $\mathrm{n}(\%)$ or mean \pm SD. '-' means data are not available. ACS, acute coronary syndrome; BMI, body mass index; CABG, coronary artery bypass grafting; Gl, gastrointestinal; HBR, high bleeding risk; HPR, high platelet reactivity; MI, myocardial infarction; NSAIDs, non-steroidal anti-inflammatory drugs; OAC, oral anticoagulant; PCI, percutaneous coronary intervention; PPI, proton pump inhibitor; SD, standard deviation; STEMI, ST-elevation myocardial infarction; TIA, transient ischemic attack.

HBR, the Chi-squared test was used to compare categorical variables, and analysis of variance was used to compare continuous variables. The cumulative incidences of thrombotic/ischemic events per JACC expert definition (or MACCE), major bleeding, and net clinical benefit per JACC expert definition (or NACE) were estimated using Kaplan-Meier methods. Hazard ratios (HR) and 95\% confidence intervals (CI) were estimated using Cox proportional hazards models, and $\mathrm{P}$ values $<0.05$ were considered to be significant. Univariate and multivariate regression analyses were conducted to determine factors associated with thrombotic/ischemic events in patients with/without HBR. Variables considered clinically important were included as covariates; these were sex, age, body weight, smoking, diabetes mellitus, presence/absence of acute coronary syndrome, history of coronary artery disease, and PRU value. Statistical analyses were performed using SAS version 9.4 (SAS Institute Inc., Cary, NC, USA).

\section{Results}

Patients

As previously reported, ${ }^{16}$ a total of 6,267 patients were enrolled in the PENDULUM Registry, of whom 3,185 (50.8\%) formed the HBR group and 3,082 (49.2\%) formed the non-HBR group according to ARC-HBR criteria. For 


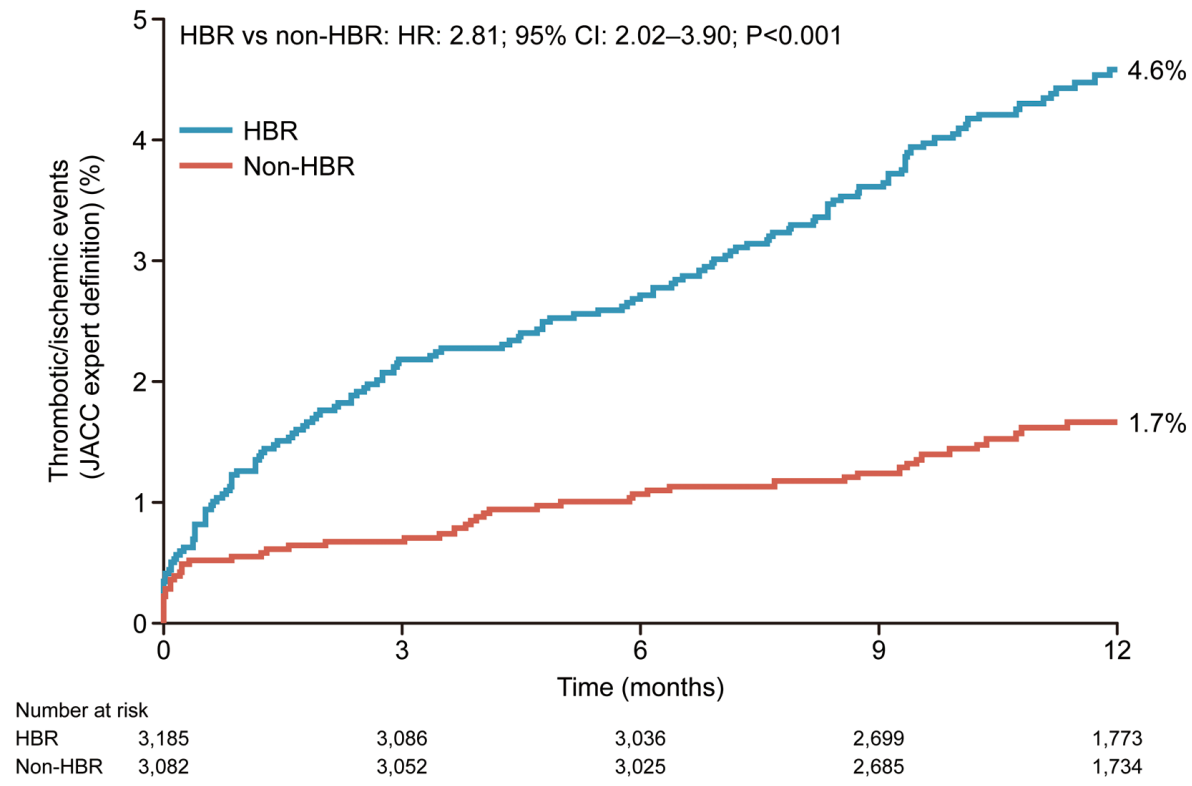

Figure 1. Cumulative incidence of thrombotic/ischemic events at 12 months after percutaneous coronary intervention according to HBR. Cl, confidence interval; HBR, high bleeding risk; HR, hazard ratio; JACC, Journal of the American College of Cardiology.

\begin{tabular}{|c|c|c|c|c|}
\hline & \multicolumn{2}{|c|}{ Event rate, $\mathrm{n}(\%)$} & \multirow[b]{2}{*}{$\mathrm{HR}(95 \% \mathrm{Cl})$} & \multirow[b]{2}{*}{$P$ value } \\
\hline & $\begin{array}{c}\text { HBR } \\
(n=3,185)\end{array}$ & $\begin{array}{l}\text { Non-HBR } \\
(n=3,082)\end{array}$ & & \\
\hline Thrombotic/ischemic events (JACC expert definition ${ }^{14}$ ) & $137(4.6)$ & $48(1.7)$ & $2.81(2.02-3.90)$ & $<0.001$ \\
\hline MACCE $^{*}$ & $202(6.8)$ & $59(2.0)$ & $3.37(2.52-4.51)$ & $<0.001$ \\
\hline Major bleeding ${ }^{\dagger}$ & $124(4.2)$ & $41(1.4)$ & $3.00(2.11-4.27)$ & $<0.001$ \\
\hline Net clinical benefit (JACC expert definition ${ }^{14}$ ) & $202(6.7)$ & $76(2.6)$ & $2.63(2.02-3.43)$ & $<0.001$ \\
\hline NACE $\ddagger$ & $292(9.7)$ & $92(3.2)$ & $3.16(2.50-3.99)$ & $<0.001$ \\
\hline
\end{tabular}

BARC, Bleeding Academic Research Consortium; Cl, confidence interval; HBR, high bleeding risk; HR, hazard ratio; JACC, Journal of the American College of Cardiology; MACCE, major adverse cardiac and cerebrovascular events; NACE, net adverse clinical events. *Includes all-cause death, non-fatal myocardial infarction, non-fatal stroke, and stent thrombosis. †BARC 3 and 5. ₹All-cause death, non-fatal myocardial infarction, non-fatal stroke, stent thrombosis, and BARC 3 and 5.

this analysis, we further stratified patients into HPR and non-HPR groups. The proportion of patients who continued to receive DAPT at 12 months was $78.3 \%$ (HBR group, 72.4\%; non-HBR group, 84.1\%) (Supplementary Figure 1), and antiplatelet treatment after DAPT was predominantly aspirin monotherapy.

Details of baseline patient characteristics for the entire population and patients with available PRU measurements are shown in Table 1. The majority of patients in all groups were male and had hypertension and hyperlipidemia. Despite the greater preponderance of male patients, no specific sex-based outcome analyses were undertaken in this study. Regardless of PRU status, patients in the HBR group were older and had a lower body mass index than those in the non-HBR group, but within each group, age and body mass index were similar regardless of HPR status. In line with patient categorization according to the ARC-HBR criteria, items such as malignancy, oral anticoagulant use at discharge, and a history of intracranial bleeding were present only in the HBR group. In both the
HBR and non-HBR groups, patients stratified as HPR were less likely to be receiving prasugrel and more likely to be receiving clopidogrel at discharge. Most other baseline characteristics were generally similar between groups.

\section{Clinical Outcomes}

The cumulative incidence of thrombotic/ischemic events at 12 months after PCI was significantly higher in the HBR group than in the non-HBR group (4.6\% vs. $1.7 \%$; HR: 2.81; 95\% CI: 2.02-3.90; P<0.001; Figure 1). The incidence of MACCE was also higher in the HBR group than in the non-HBR group (6.8\% vs. 2.0\%; Supplementary Figure 2). Event component rates at 12 months after PCI are shown in Supplementary Table 2. Cardiovascular death accounted for approximately half of the events for patients with thrombotic/ischemic events, followed by MI and stroke.

The incidence of major bleeding at 12 months after PCI was significantly higher in HBR patients than non-HBR patients (Table 2). The cumulative incidence of net clinical benefit and NACE are also shown in Table 2. 
A

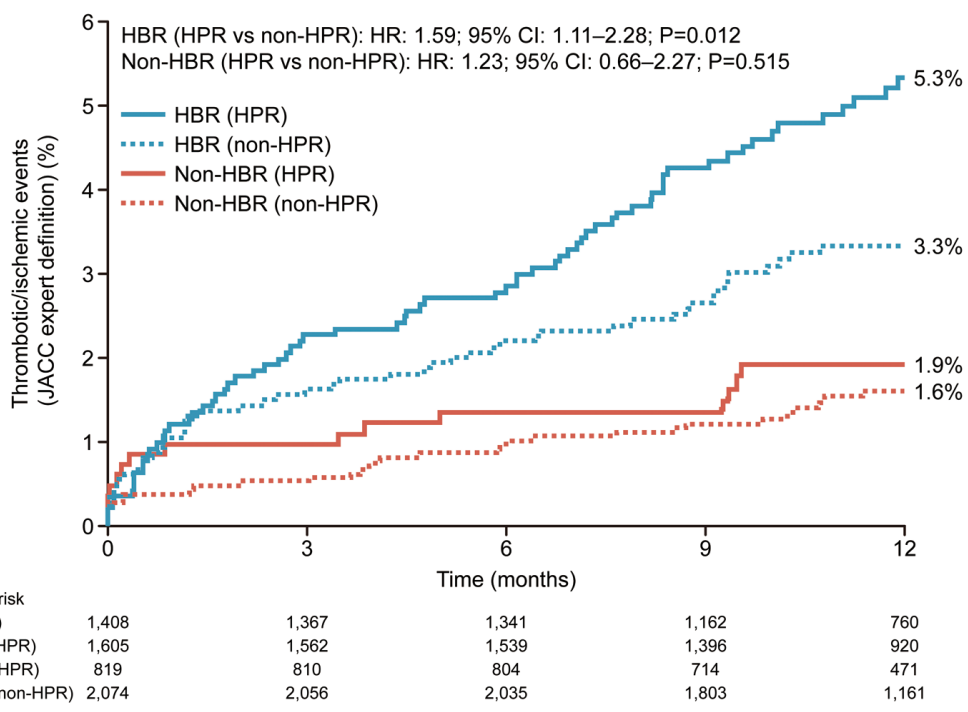

Number at risk HBR (HPR)

HBR (non-HPR)

Non-HBR (HPR)

2,074

2,056

2,035

1,803

B

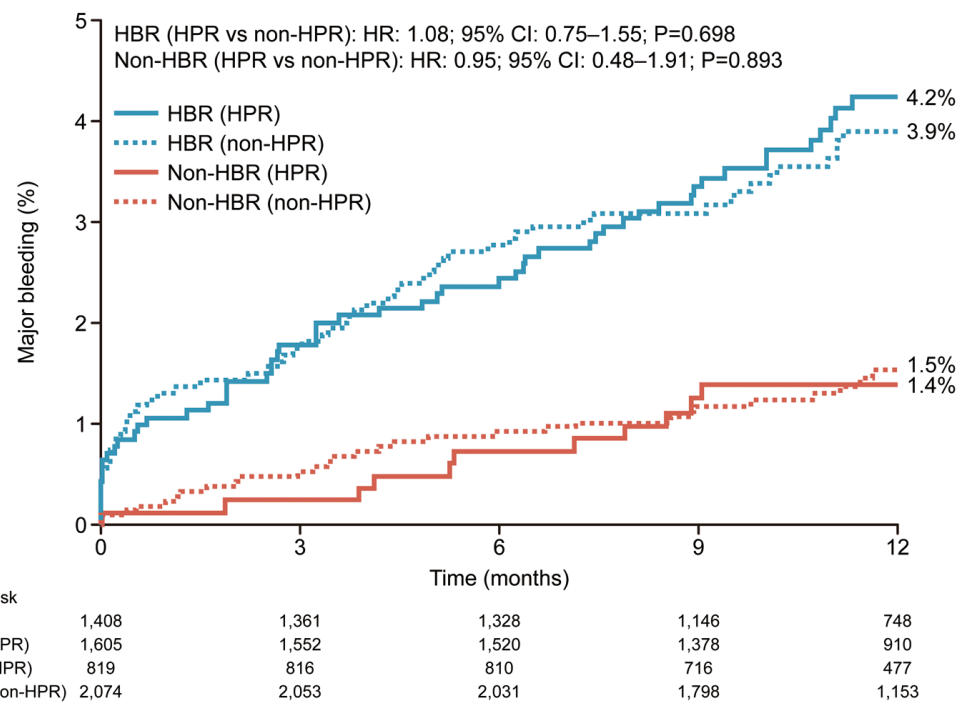

Number at risk

HBR (HPR)

HBR (non-HPR)

Non-HBR (HPR)

819
2,074

C

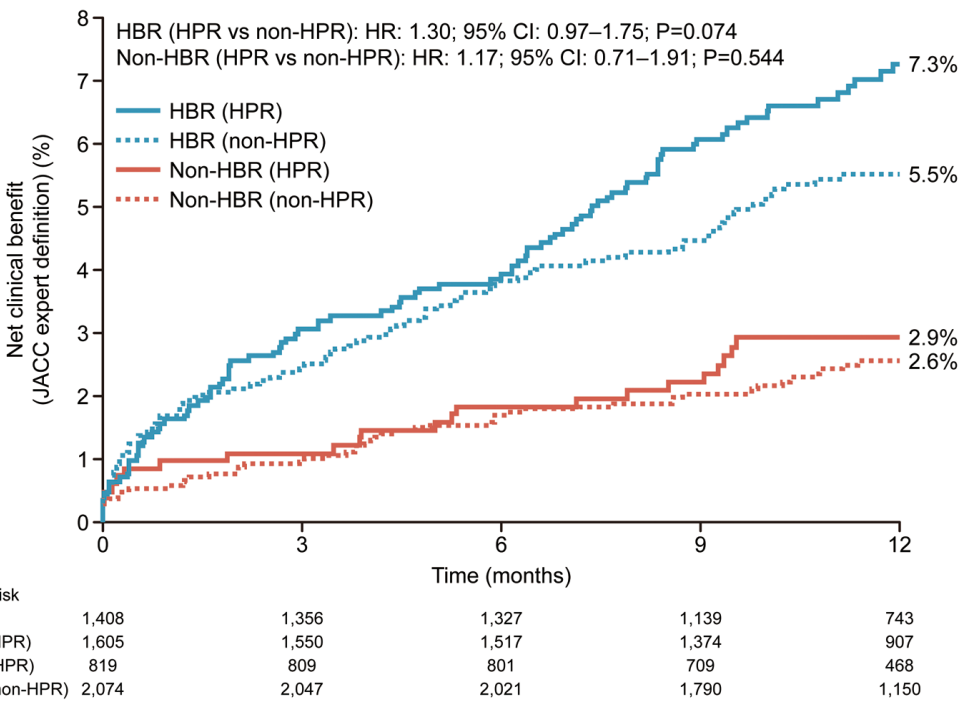

Figure 2. Cumulative incidence of (A) thrombotic/ischemic events (JACC Expert Definition ${ }^{14}$ ), (B) major bleeding, and (C) net clinical benefit (JACC Expert Definition ${ }^{14}$ ) at 12 months after percutaneous coronary intervention according to HBR. Cl, confidence interval; HBR, high bleeding risk; HPR, high platelet reactivity; HR, hazard ratio; JACC, Journal of the American College of Cardiology. 


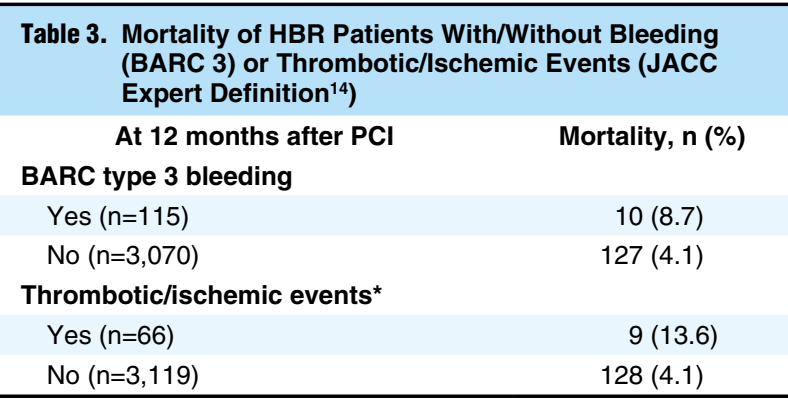

Abbreviations as in Tables 1,2. *Excludes cardiovascular death.

The cumulative incidences of thrombotic/ischemic events, major bleeding, and net clinical benefit by HPR status are shown in Figure 2. In HBR patients, thrombotic/ischemic events were statistically significantly more frequent in the HPR subgroup than in the non-HPR subgroup (HR: 1.59; $95 \%$ CI: 1.11-2.28; $\mathrm{P}=0.012$ ). The cumulative incidence of MACCE and NACE was numerically higher in patients with HPR (Supplementary Figure 3). In addition, the cumulative incidence of thrombotic/ischemic events and major bleeding stratified by patients on prasugrel or clopidogrel at discharge is shown in Supplementary Figure 4. The cumulative incidence of thrombotic/ischemic events was higher in the HPR patients than non-HPR patients, regardless of the medication at discharge.

Table 3 reports mortality at 12 months after PCI. In HBR patients with and without BARC type 3 bleeding, $8.7 \%$ and $4.1 \%$, respectively, were reported to have died at 12 months. In HBR patients with and without thrombotic/ ischemic events, $13.6 \%$ and $4.1 \%$, respectively, had died 12 months after PCI. Overall, HBR patients with bleeding or thrombotic/ischemic events had a higher incidence of death.
Table 4 summarizes the multivariate Cox regression analysis for thrombotic/ischemic events in HBR patients and non-HBR patients. After adjustment of covariates, thrombotic/ischemic events were found to be associated with PRU values in HBR patients, but not in non-HBR patients.

\section{Discussion}

A clear understanding of ischemic risk and mortality rates in HBR patients is critical when choosing antithrombotic treatment for patients undergoing PCI, but pertinent data are lacking. In this post hoc analysis of patients in the PENDULUM Registry, who underwent PCI via contemporary methods, we found that HBR patients had a higher incidence of ischemic events (2.8-fold increase) and bleeding events (3-fold increase) compared with non-HBR patients. Using the JACC expert definitions, ${ }^{14}$ the cumulative incidence of thrombotic/ischemic events in HBR patients was significantly higher in the HPR group than in the nonHPR group. However, there was no difference between HPR patients and non-HPR patients in the incidence of bleeding events. Among non-HBR patients, HPR and non-HPR subgroups showed a disassociation in thrombotic/ischemic event rates from an early stage, suggesting a relationship between early platelet function and ischemic event. However, among HBR patients, the event curves for HPR and non-HPR subgroups overlapped at the beginning. In addition, DAPT was almost continuous and there was no switch from prasugrel to clopidogrel. Therefore, patient background factors, including the higher risk factors in HBR patients, may have contributed to this finding.

\section{Thrombotic/lschemic Events: Overlap and Effect on Mortality}

In this study, thrombotic events were higher in the HBR group than in the non-HBR group, and rates of bleeding

\begin{tabular}{|c|c|c|c|c|}
\hline & $\mathbf{n}$ & Events, n (\%) & HR $(95 \% \mathrm{Cl})$ & $P$ value \\
\hline \multicolumn{5}{|l|}{ HBR } \\
\hline Sex: male vs. female & 2,332 vs. 853 & $102(4.4)$ vs. 35 (4.1) & $1.15(0.70-1.88)$ & 0.586 \\
\hline Age: $\geq 75$ vs. $<75$ years & 1,924 vs. 1,261 & $84(4.4)$ vs. 53 (4.2) & $0.98(0.64-1.51)$ & 0.940 \\
\hline Body weight: $\leq 50$ vs. $>50 \mathrm{~kg}$ & 583 vs. 2,528 & 37 (6.3) vs. 96 (3.8) & $1.60(0.95-2.69)$ & 0.079 \\
\hline Smoking: yes vs. no* & 595 vs. 2,217 & 27 (4.5) vs. $86(3.9)$ & $1.24(0.77-2.00)$ & 0.385 \\
\hline Diabetes mellitus: yes vs. no & 1,515 vs. 1,670 & $81(5.3)$ vs. $56(3.4)$ & $1.84(1.22-2.79)$ & 0.004 \\
\hline ACS: ACS vs. non-ACS & 923 vs. 2,262 & 57 (6.2) vs. 80 (3.5) & $1.81(1.19-2.76)$ & 0.006 \\
\hline History of CAD-related conditions: yes vs. no & 1,561 vs. 1,624 & $68(4.4)$ vs. 69 (4.2) & $1.24(0.83-1.87)$ & 0.294 \\
\hline PRU: $>208$ vs. $\leq 208$ & 1,408 vs. 1,605 & $70(5.0)$ vs. 51 (3.2) & $1.69(1.13-2.54)$ & 0.011 \\
\hline \multicolumn{5}{|l|}{ Non-HBR } \\
\hline Sex: male vs. female & 2,577 vs. 505 & $39(1.5)$ vs. $9(1.8)$ & $1.35(0.42-4.32)$ & 0.609 \\
\hline Age: $\geq 75$ vs. $<75$ years & 400 vs. 2,682 & $6(1.5)$ vs. $42(1.6)$ & $0.22(0.03-1.64)$ & 0.138 \\
\hline Body weight: $\leq 50$ vs. $>50 \mathrm{~kg}$ & 211 vs. 2,798 & 4 (1.9) vs. $40(1.4)$ & $2.00(0.54-7.36)$ & 0.298 \\
\hline Smoking: yes vs. no* & 1,079 vs. 1,734 & 21 (1.9) vs. 20 (1.2) & $1.25(0.63-2.49)$ & 0.520 \\
\hline Diabetes mellitus: yes vs. no & 1,252 vs. 1,830 & $16(1.3)$ vs. $32(1.7)$ & $0.58(0.28-1.21)$ & 0.144 \\
\hline ACS: ACS vs. non-ACS & 1,092 vs. 1,990 & $22(2.0)$ vs. $26(1.3)$ & $2.21(1.07-4.58)$ & 0.032 \\
\hline History of CAD-related conditions: yes vs. no & 1,337 vs. 1,745 & $22(1.6)$ vs. 26 (1.5) & $1.32(0.63-2.77)$ & 0.457 \\
\hline PRU: $>208$ vs. $\leq 208$ & 819 vs. 2,074 & $15(1.8)$ vs. $31(1.5)$ & $1.56(0.78-3.11)$ & 0.210 \\
\hline
\end{tabular}

CAD, coronary artery disease; PRU, P2Y 12 reaction unit. Other abbreviations as in Tables 1,2. *'Yes' includes current smokers or those smoking within the past 2 years; 'no' includes those who have never smoked or those who ceased smoking $>2$ years previously. 
events were also higher in the HBR group compared with the non-HBR group. This is unsurprising as the ARCHBR criteria include items that have long been known as thrombotic risk factors, such as anemia, chronic kidney disease, advanced age, and cerebral infarction. ${ }^{6}$ Furthermore, these overlapping factors are the most frequent criteria for identification of HBR in daily practice. ${ }^{7}$ Indeed, both MACCE and major bleeding rates in HBR patients in the present study were comparable with a previous report evaluating the ARC-HBR criteria in real-world patients undergoing PCI. ${ }^{\mathbf{2}}$

Notably, our data have shown that the risk of death after non-fatal ischemic events was numerically higher than the risk of death after bleeding complications. This finding is in line with a stratified analysis of 4-stent post-approval studies, in which it was shown that major bleeding and ischemic events had a similar effect on the subsequent risk of mortality in both HBR and non-HBR groups. ${ }^{19}$ Additionally, the current data are consistent with a retrospective cohort study about mortality following PCI, in which bleeding and MI were found to be associated with comparable mortality risks during follow up. ${ }^{20}$

The optimal antithrombotic treatment to manage bleeding in patients with HBR has been a point of considerable debate in recent years. However, considering the equally high ischemic risk in this patient group and the effect of ischemic events on death, it is clear that careful consideration should be made regarding the use of antithrombotic therapy in this population. For patients at high risk of both bleeding and ischemia, treatment strategies that reduce bleeding while simultaneously not increasing the risk of ischemia are recommended. ${ }^{21}$

\section{Platelet Reactivity as a Risk Factor for Thrombotic Events}

In this post hoc analysis, HPR was an independent risk factor for thrombotic events in patients with HBR. This trend was not seen in the non-HBR group. When thrombotic events were evaluated using both the traditional and JACC expert definitions, we found that HPR was statistically non-significant in the traditional MACCE evaluation, but was significant in the JACC expert definition of thrombotic/ischemic events. This may reflect the fact that the JACC expert definition does not include bleeding events, which allows for more accurate estimation of thrombotic/ischemic events. Thus, increased clarity in categorizing and evaluating thrombotic events may allow for a more appropriate assessment of the balance between bleeding and thrombotic events in patients with HBR. In addition, non-HPR status did not increase the frequency of bleeding events in HBR patients.

We have previously reported that post-PCI PRU levels were associated with the occurrence of MACCE 12 months after PCI. ${ }^{15}$ The current post hoc analysis results further reinforce this concept, especially within the subpopulation of HBR patients. Based on the current data, we can hypothesize that adequately lowering PRU with a potent antiplatelet agent (such as prasugrel), even in HBR patients, may reduce thrombotic events without increasing bleeding events. However, because there was no significant difference in net clinical benefit, the true effect of lowering PRU requires prospective evaluation in randomized, controlled trials.

\section{Common Risk Factors and Management}

Management of patients with common risk factor(s) for bleeding and thrombosis will increasingly play an important role in the era of personalized medicine because the prevalence of HBR is high in daily cardiology practice. Several approaches have been proposed to address this challenging clinical scenario. One is the de-escalation of antithrombotic therapy, starting with potent antiplatelet therapy followed by a decrease in the potency. ${ }^{22-24}$ The other is escalation, starting with a less potent antiplatelet agent, followed by adjustment so that the full effect of the antiplatelet agent is achieved.25-27 As the present study showed that HPR was an independent predictor of ischemic events in patients with HBR receiving long-term DAPT, the importance of HPR in HBR patients receiving short durations of DAPT treatment must also be considered. Although de-escalation of antiplatelet therapy for patients with HBR is possible, simple downward tapering may be risky due to the possibility of ischemic event occurrence. One possible strategy is to prescribe short-term DAPT and a potent single-agent $\mathrm{P} 2 \mathrm{Y}_{12}$ inhibitor, which should reduce the bleeding risk while maintaining low ischemic risk. Recent trials have shown the potential of this strategy. ${ }^{28}$ However, it must be noted that there are no studies specifically comparing different antithrombotic strategies in HBR patients, and definitive evidence for the optimal treatment strategy in HBR patients is required. Nevertheless, we consider that the data from our current study may provide a useful foundation for future studies exploring this complex but common clinical scenario.

\section{Study Limitations}

Limitations of this analysis include its post hoc nature, meaning that not all variables conforming to ARC-HBR criteria were collected. As East Asian and Western patients are reported to have different risk profiles for bleeding and thrombosis, ${ }^{29}$ the risk of bleeding may be unduly emphasized in our data. However, the risk of ischemic and bleeding events in the HBR group compared with the non-HBR group was similar to previously reported data from studies outside of Japan. ${ }^{\mathbf{7}}$ Further studies will be required to clarify this point. In addition, this was a retrospective analysis of prospectively collected registry data. It is possible that the presence of unmeasured factors may have confounded the multivariate analyses. It should also be noted that although this study was registered before the concept of HBR was established, selection bias due to drug preference cannot be denied. Another limitation was that only a single type of antiplatelet test was used, and the timing of PRU measurement was limited to immediately after PCI, potentially restricting the generalizability of the resulting data. Although the dose of antiplatelet medication prescribed differed from that used in other countries, the PRU cut-off value of 208 is a universal definition, and we believe that the concepts presented in this study are applicable worldwide. Most patients were treated with DAPT over a long duration, which may not align with current recommendations for reduced treatment periods in high-risk patients. Importantly, the findings of this study do not support any particular duration of DAPT in HBR patients with HPR. Finally, as PRU values were only measured at one timepoint (12 or $48 \mathrm{~h}$ after PCI), whether the PRU values measured in the acute phase were associated with long-term clinical outcomes at 12 months was not determined. However, the relationship between PRU immediately after PCI and outcomes in our analysis is supported by the following points: (1) similar studies such as ADAPT-DES and the 
Japanese PRASFIT-ACS and J-CHIPS studies showed an association between PRU and outcomes;30-32 (2) DAPT continuation in our study was high at $70-80 \%$, and no treatment changes had been made; (3) patients received a loading dose or continuous administration for $\geq 2$ weeks; and (4) the association between stent thrombosis and MI and PRU was confirmed by receiver operating characteristics analysis at $\sim 0.7$ by $\mathrm{C}$-statistics despite moderating. ${ }^{16}$

\section{Conclusions}

In conclusion, the incidence of thrombotic/ischemic events in this analysis was approximately 3 -fold higher in HBR patients than in non-HBR patients. In HBR patients, HPR was associated with a significantly higher incidence of thrombotic/ischemic events, but not bleeding events. These results suggest that adequate control of platelet reactivity levels is essential, even in patients at high risk of bleeding.

\section{Acknowledgment}

The authors thank Edanz (www.edanz.com) for providing medical writing support, which was funded by Daiichi Sankyo Co., Ltd.

\section{Sources of Funding}

This study was supported by Daiichi Sankyo Co., Ltd., Tokyo, Japan. Daiichi Sankyo Co., Ltd. played a role in the design and conduct of the study; collection, management, and interpretation of the data; preparation, review, or approval of the manuscript; and decision to submit the manuscript for publication.

\section{Disclosures}

K. Kadota has received remuneration from Daiichi Sankyo Co., Ltd., and Sanofi K.K. K. Nakao has received remuneration from Daiichi Sankyo Co., Ltd. Y. Nakagawa has received remuneration from Bristol Myers Squibb K.K., Kowa Pharmaceutical Co., Ltd., Daiichi Sankyo Co., Ltd., Bayer Yakuhin Ltd., Sanofi K.K., Boston Scientific Corporation, and Abbott Medical Japan LLC., and research funding from Daiichi Sankyo Co., Ltd., Bayer Yakuhin Ltd., Sanofi K.K., Boston Scientific Corporation, and Abbott Medical Japan LLC. J. Shite has received remuneration from Daiichi Sankyo Co., Ltd., Nipro Corporation, Abbott Japan LLC, and Terumo Corporation. H. Yokoi has received remuneration and scholarship funds or donations from Daiichi Sankyo Co., Ltd. K. Kozuma has received remuneration and research funding from Daiichi Sankyo Co., Ltd. K. Tanabe has received remuneration from Daiichi Sankyo Co., Ltd., Sanofi K.K., AstraZeneca K.K., Abbott Medical Japan LLC., Boston Scientific Corporation, and Terumo Corporation. T. Akasaka has received remuneration from Abbott Medical Japan LLC., and Otsuka Pharmaceutical Co., Ltd., research funding from Daiichi Sankyo Co., Ltd., scholarship funds or donations from Abbott Medical Japan LLC., Nipro Corporation, and Terumo Corporation, and has a personal relationship with Terumo Corporation. T. Shinke has received remuneration and research funding from Daiichi Sankyo Co., Ltd., Bayer Yakuhin Ltd., Bristol-Myers Squibb K.K., and Nippon Boehringer Ingelheim Co., Ltd. T. Ueno received consultancy fees from Japan Medical Device Technology Co., Ltd., and Nipro Corporation. A. Hirayama has received remuneration from Daiichi Sankyo Co., Ltd., Bayer Yakuhin Ltd., and Takeda Pharmaceutical Co., Ltd. S. Uemura has received remuneration from Daiichi Sankyo Co., Ltd., Nippon Boehringer Ingelheim Co., Ltd., Amgen Astellas BioPharma Co., Ltd., Abbott Medical Japan LLC., Sanofi K.K., Terumo Corporation, and Bayer Yakuhin Ltd.; and research funding from Daiichi Sankyo Co., Ltd.; and scholarship funds or donations from Daiichi Sankyo Co., Ltd., Astellas Pharma Inc., Otsuka Pharmaceutical Co., Ltd., Goodman Co., Ltd., Shionogi Inc., Sumitomo Dainippon Pharma Co., Ltd., Boston Scientific Japan K.K., Kaken Pharmaceutical Co., Ltd., Takeda Pharmaceutical Co., Ltd., Taisho Pharmaceutical Co., Ltd., Mitsubishi Tanabe Pharmaceutical Co., Ltd., Japan Lifeline Co., Ltd., MSD K.K., Nipro Corporation, Actelion Pharmaceuticals Japan Ltd., Pfizer Japan Inc., Abbott Medical Japan LLC., Sanofi K.K., Terumo Corporation, and Bayer Yakuhin Ltd. A. Harada, T. Kuroda, and A. Takita are employees of Daiichi Sankyo Co., Ltd. Y. Murakami has received remuneration from SRD Co., Ltd. S. Saito has received consultancy fees from Japan Lifeline Inc., and Terumo Corporation; and remuneration from Daiichi Sankyo Co., Ltd, Abbott Medical Japan LLC., Boston Scientific Corporation, and Medtronic Japan Co., Ltd. M. Nakamura has received remuneration from Daiichi Sankyo Co., Ltd., Sanofi K.K., Terumo Corporation, and Bristol Myers Squibb K.K, and research funding from Daiichi Sankyo Co., Ltd., Sanofi K.K., and Bayer Yakuhin K.K. R. Iijima has no conflicts of interest to declare.

T. Ueno, A. Hirayama, and S. Uemura are members of Circulation Journal's Editorial Team.

\section{Data Availability}

The deidentified participant data and related study documents will be shared on request. The data request will be accepted for up to 36 months after the publication of this article. Requests should be made to the corresponding author and researchers who make the request should include a methodologically sound proposal on how the data will be used. The proposal may be reviewed for approval by the responsible personnel at Daiichi Sankyo Co. Ltd., and the data requestors will need to sign a data access agreement. The data will be shared in an appropriate way depending on the type of data requested.

\section{References}

1. Moon JY, Franchi F, Rollini F, Angiolillo DJ. Evolution of coronary stent technology and implications for duration of dual antiplatelet therapy. Prog Cardiovasc Dis 2018; 60: 478-490.

2. Mangieri A, Gallo F, Sticchi A, Khokhar AA, Laricchia A, Giannini F, et al. Dual antiplatelet therapy in coronary artery disease: From the past to the future prospective. Cardiovasc Interv Ther 2020; 35: 117-129.

3. Iijima R, Nakamura M. Optimal revascularization strategy for acute coronary syndromes with high bleeding risk: It is hard to please all parties. Circ J 2021; 85: 1942-1943.

4. Esmonde S, Sharma D, Peace A. Antiplatelet agents in uncertain clinical scenarios: A bleeding nightmare. Cardiovasc Diagn Ther 2018; 8: 647-662.

5. Saito Y, Kobayashi Y. Contemporary coronary drug-eluting and coated stents: A mini-review. Cardiovasc Interv Ther 2021; 36: $20-22$.

6. Urban P, Mehran R, Colleran R, Angiolillo DJ, Byrne RA, Capodanno $\mathrm{D}$, et al. Defining high bleeding risk in patients undergoing percutaneous coronary intervention: A consensus document from the Academic Research Consortium for High Bleeding Risk. Eur Heart J 2019; 40: 2632-2653.

7. Cao D, Mehran R, Dangas G, Baber U, Sartori S, Chandiramani $\mathrm{R}$, et al. Validation of the Academic Research Consortium High Bleeding Risk definition in contemporary PCI patients. $J$ Am Coll Cardiol 2020; 75: 2711-2722.

8. Ueki Y, Bär S, Losdat S, Otsuka T, Zanchin C, Zanchin T, et al. Validation of the Academic Research Consortium for High Bleeding Risk (ARC-HBR) criteria in patients undergoing percutaneous coronary intervention and comparison with contemporary bleeding risk scores. EuroIntervention 2020; 16: 371-379.

9. Baber U, Mehran R, Giustino G, Cohen DJ, Henry TD, Sartori $\mathrm{S}$, et al. Coronary thrombosis and major bleeding after PCI with drug-eluting stents: Risk scores from PARIS. J Am Coll Cardiol 2016; 67: 2224-2234.

10. Mauri L, Kereiakes DJ, Yeh RW, Driscoll-Shempp P, Cutlip DE, Steg G, et al. Twelve or 30 months of dual antiplatelet therapy after drug-eluting stents. $N$ Engl J Med 2014; 371: 2155-2166.

11. Natsuaki M, Morimoto T, Yamaji K, Watanabe H, Yoshikawa $\mathrm{Y}$, Shiomi H, et al. Prediction of thrombotic and bleeding events after percutaneous coronary intervention: CREDO-Kyoto thrombotic and bleeding risk scores. J Am Heart Assoc 2018; 7: e008708.

12. Yeh RW, Secemsky EA, Kereiakes DJ, Normand SLT, Gershlick $\mathrm{GH}$, Cohen DJ, et al. Development and validation of a prediction rule for benefit and harm of dual antiplatelet therapy beyond 1 year after percutaneous coronary intervention. JAMA 2016; 315: $1735-1749$.

13. Galli M, Benenati S, Capodanno D, Franchi F, Rollini F, D'Amario D, et al. Guided versus standard antiplatelet therapy in patients undergoing percutaneous coronary intervention: A systematic review and meta-analysis. Lancet 2021; 397: 14701483.

14. Capodanno D, Morice MC, Angiolillo DJ, Bhatt DL, Byrne RA, Colleran R, et al. Trial design principles for patients at high bleeding risk undergoing PCI: JACC scientific expert panel. $J$ 
Am Coll Cardiol 2020; 76: 1468-1483.

15. Nakamura M, Kadota K, Takahashi A, Kanda J, Anzai H, Ishii Y, et al. Relationship between platelet reactivity and ischemic and bleeding events after percutaneous coronary intervention in East Asian patients: 1-Year results of the PENDULUM registry. J Am Heart Assoc 2020; 9: e015439.

16. Nakamura M, Kadota K, Nakao K, Nakagawa Y, Shite J, Yokoi $\mathrm{H}$, et al. High bleeding risk and clinical outcomes in East Asian patients undergoing percutaneous coronary intervention: The PENDULUM registry. EuroIntervention 2021; 16: 1154-1162.

17. Mehran R, Rao SV, Bhatt DL, Gibson CM, Caixeta A, Eikelboom $\mathrm{J}$, et al. Standardized bleeding definitions for cardiovascular clinical trials: A consensus report from the Bleeding Academic Research Consortium. Circulation 2011; 123: 2736-2747.

18. Giustino G, Redfors B, Kirtane AJ, Mehran R, Dangas GD, Witzenbichler B, et al. Platelet reactivity and risk of ischemic stroke after coronary drug-eluting stent implantation: From the ADAPT-DES study. JACC Cardiovasc Interv 2018; 11: 1277-1286.

19. Sorrentino S, Claessen BE, Chandiramani R, Guedeney P, Vogel $\mathrm{B}, \mathrm{Baber} \mathrm{U}$, et al. Long-term safety and efficacy of durable polymer cobalt-chromium everolimus-eluting stents in patients at high bleeding risk: A patient-level stratified analysis from four postapproval studies. Circulation 2020; 141: 891-901.

20. Kazi DS, Leong TK, Chang TI, Solomon MD, Hlatky MA, Go AS. Association of spontaneous bleeding and myocardial infarction with long-term mortality after percutaneous coronary intervention. J Am Coll Cardiol 2015; 65: 1411-1420.

21. Giustino G, Costa F. Characterization of the individual patient risk after percutaneous coronary intervention: At the crossroads of bleeding and thrombosis. JACC Cardiovasc Interv 2019; 12: $831-834$.

22. Claassens DMF, Vos GJA, Bergmeijer TO, Hermanides R, van't Hof AWJ, van der Harst P, et al. A genotype-guided strategy for oral P2Y 12 inhibitors in primary PCI. N Engl J Med 2019; 381: $1621-1631$.

23. Cuisset T, Deharo P, Quilici J, Johnson TW, Deffarges S, Bassez $\mathrm{C}$, et al. Benefit of switching dual antiplatelet therapy after acute coronary syndrome: The TOPIC (timing of platelet inhibition after acute coronary syndrome) randomized study. Eur Heart $J$ 2017; 38: 3070-3078.

24. Sibbing D, Aradi D, Jacobshagen C, Gross L, Trenk D, Geisler $\mathrm{T}$, et al. Guided de-escalation of antiplatelet treatment in patients with acute coronary syndrome undergoing percutaneous coronary intervention (TROPICAL-ACS): A randomised, open- label, multicentre trial. Lancet 2017; 390: 1747-1757.

25. Pereira NL, Farkouh ME, So D, Lennon R, Geller N, Mathew $\mathrm{V}$, et al. Effect of genotype-guided oral P2Y12 inhibitor selection vs conventional clopidogrel therapy on ischemic outcomes after percutaneous coronary intervention: The TAILOR-PCI randomized clinical trial. JAMA 2020; 324: $761-771$.

26. Price MJ, Berger PB, Teirstein PS, Tanguay JF, Angiolillo DJ, Spriggs D, et al. Standard- vs high-dose clopidogrel based on platelet function testing after percutaneous coronary intervention: The GRAVITAS randomized trial. JAMA 2011; 305: 1097-1105.

27. Pereira NL, Rihal C, Lennon R, Marcus G, Shrivastava S, Bell $\mathrm{MR}$, et al. Effect of CYP2C19 genotype on ischemic outcomes during oral $\mathrm{P}_{2} \mathrm{Y}_{12}$ inhibitor therapy: A meta-analysis. JACC Cardiovasc Interv 2021; 14: 739-750.

28. Nakamura M, Kadota K, Nakao K, Nakagawa Y, Shite J, Yokoi H, et al. Single antiplatelet therapy with prasugrel vs. dual antiplatelet therapy in Japanese percutaneous coronary intervention patients with high bleeding risk. Circ $J$ 2021; 85: 785-793.

29. Levine GN, Jeong YH, Goto S, Anderson JL, Huo Y, Mega JL, et al. Expert consensus document: World Heart Federation expert consensus statement on antiplatelet therapy in East Asian patients with ACS or undergoing PCI. Nat Rev Cardiol 2014; 11: 597-606.

30. Nakamura M, Isshiki T, Kimura T, Ogawa H, Yokoi H, Nanto $\mathrm{S}$, et al. Optimal cutoff value of $\mathrm{P} 2 \mathrm{Y} 12$ reaction units to prevent major adverse cardiovascular events in the acute periprocedural period: Post-hoc analysis of the randomized PRASFIT-ACS study. Int J Cardiol 2015; 182: 541-548.

31. Stone GW, Witzenbichler B, Weisz G, Rinaldi MJ, Neumann FJ, Metzger DC, et al. Platelet reactivity and clinical outcomes after coronary artery implantation of drug-eluting stents (ADAPTDES): A prospective multicentre registry study. Lancet 2013; 382: $614-623$.

32. Nishikawa M, Takeda $Y$, Isomura N, Tanigawa T, Nanasato $\mathrm{M}$, Tsukahara K, et al. Association between high platelet reactivity following dual antiplatelet therapy and ischemic events in Japanese patients with coronary artery disease undergoing stent implantation. J Atheroscler Thromb 2020; 27: 13-24.

\section{Supplementary Files}

Please find supplementary file(s);

http://dx.doi.org/10.1253/circj.CJ-21-0717 